\title{
Characterization of pseudosingle bunch kick-and-cancel operational mode
}

\author{
C. Sun, ${ }^{*}$ D. S. Robin, C. Steier, and G. Portmann \\ Lawrence Berkeley National Laboratory, Advanced Light Source, Berkeley, California 94720-8228, USA
}

(Received 30 September 2015; published 18 December 2015)

\begin{abstract}
Pseudosingle-bunch kick-and-cancel (PSB-KAC) is a new operational mode at the Advanced Light Source of Lawrence Berkeley National Laboratory that provides full timing and repetition rate control for single x-ray pulse users while being fully transparent to other users of synchrotron radiation light. In this operational mode, a single electron bunch is periodically displaced from a main bunch train by a fast kicker magnet with a user-on-demand repetition rate, creating a single $\mathrm{x}$-ray pulse to be matched to a typical laser excitation pulse rate. This operational mode can significantly improve the signal to noise ratio of single $\mathrm{x}$-ray pulse experiments and drastically reduce dose-induced sample damage rate. It greatly expands the capabilities of synchrotron light sources to carry out dynamics and time-of-flight experiments. In this paper, we carry out extensive characterizations of this PSB-KAC mode both numerically and experimentally. This includes the working principle of this mode, resonance conditions and beam stability, experimental setups, and diagnostic tools and measurements.
\end{abstract}

DOI: 10.1103/PhysRevSTAB.18.120702

PACS numbers: 29.20.db, 41.85.-p

\section{INTRODUCTION}

Pseudosingle-bunch kick-and-cancel (PSB-KAC) operational mode has been successfully brought into user operations [1-5] at the Advanced Light Source (ALS) of Lawrence Berkeley National Laboratory. In this operational mode, a single electron bunch is transversely displaced from a main electron bunch train circulating in the ALS storage ring by a fast kicker magnet. Beam users can use the radiation light emitted from the kicked single bunch by blocking the light from the main bunch train, thus allowing to run single bunch experiments during the multibunch operation. By choosing the right kicker pulse pattern and storage ring lattice, the single bunch can be periodically displaced from the main bunch train with a user-on-demand repetition rate, creating a single $\mathrm{x}$-ray pulse to be matched to a typical laser excitation pulse rate. Thus, it can significantly improve the signal to noise ratio and drastically reduce dose-induced sample damage rate for single bunch experiments. This operation mode greatly expands the capabilities of synchrotron light sources to carry out dynamics and time-of-flight experiments.

The performance and advantage of this PSB-KAC operational mode have been reported in our previous works [1-5]. Now with several years of operation and exploration of PSB we have a solid understanding of the conditions under which PSB works well and those were there issues. In this paper, we will present our extensive studies and

\section{*CCSun@lbl.gov}

Published by the American Physical Society under the terms of the Creative Commons Attribution 3.0 License. Further distribution of this work must maintain attribution to the author $(s)$ and the published article's title, journal citation, and DOI. characterizations of this PSB-KAC mode. In Sec. II, we will study the working principle of this PSB-KAC mode, including the closed orbit, beam resonance conditions, and stability issues. In Sec. III, we will discuss the setups of the storage ring and kicker timing for the KAC operation. In Sec. IV, we will present the experimental results and diagnostics tools. The paper is concluded in Sec. V.

\section{PSEUDOSINGLE BUNCH KICK-AND-CANCEL SCHEME}

\section{A. Camshaft kicker}

Before reviewing our results for the pseudosingle bunch kick-and-cancel scheme, let us first give a brief introduction of our single bunch (camshaft) kicker. It is a half-meter long strip-line kicker [6] and installed at the sector 2 straight in the ALS storage ring as shown in Fig. 4. This kicker has the repetition rate as high as the ALS storage ring revolution rate of $1.5 \mathrm{MHz}$, capable of kicking the beam every turn. It also has a short pulse duration about $70 \mathrm{~ns}$ in the full width, capable of only kicking the camshaft bunch filled in the ion-cleaning gap without kicking the main bunch train. The maximum kick angle that the kicker can deliver is about $73 \mu \mathrm{rad}$ for $1.9 \mathrm{GeV}$ electron beam. The beam is kicked vertically since the vertical beam size and angle divergence are much smaller than the horizontal ones and the same kick angle will result in a much larger separation in terms of the beam size than the kick in the horizontal plane.

The camshaft kicker was originally designed to displace the camshaft bunch to a permanent closed orbit for a PSB operation. Operationally, there are a number of restrictions on the kicker pulser. Neither the kick angle nor kick polarity can be changed quickly. However, the option of variable kick frequency and kick pattern exists. For 
instance, the kicker can be adjusted to kick every turn, every $n$th turn, or even has an uneven kick pattern such as kicking once, then kicking two turns later, waiting $1 \mathrm{~ms}$ and repeating. This turns out to be an important feature to operate this kicker in the kick-and-cancel scheme.

\section{B. Principle of KAC scheme}

The working principle of the PSB-KAC scheme is to use the camshaft kicker to displace a single bunch to a different orbit and then kick it back to its original orbit within a few turns by adjusting the machine tune and the kick pattern. This idea was originated from a Memorandum of Brookhaven National Laboratory in 1980s [7]. In this memorandum, a ferrite magnet, so-called "wobbler," was proposed to kick the electron beam. The kick amplitude of this wobbler was adjustable. Therefore, a sequence of kicks with different amplitudes was used to first kick the electron beam out of the design orbit and then to kick it back to the design orbit and there was no requirement on the machine tunes. However, at the ALS the kick amplitude cannot be quickly adjusted, therefore the machine tune must have a right value in order to restore the displaced beam with the same kick amplitude.

Mathematically, the KAC scheme can be understood as follows. Assuming that a camshaft bunch is kicked at the $i$ th turn with a kick angle of $\theta_{y}$, the orbit offset and angle at the $n$th turn due to this kick are given by

$$
\begin{aligned}
& y_{n}^{i}=\theta_{y} \beta_{y} \sin \left[2 \pi(n-i) \nu_{y}\right], \\
& y_{n}^{\prime i}=\theta_{y}\left\{\cos \left[2 \pi(n-i) \nu_{y}\right]-\alpha_{y} \sin \left[2 \pi(n-i) \nu_{y}\right]\right\},
\end{aligned}
$$

where $\beta_{y}$ and $\alpha_{y}$ are Twiss functions of the machine at the kicker location and $\nu_{y}$ is the vertical tune of the machine. To restore the displaced orbit after the $n$th turn, the superpositions of orbit offsets and angles from each kick need to be zero, i.e.,

$$
\sum_{i=0}^{n} y_{n}^{i}=0, \quad \sum_{i=0}^{n} y_{n}^{\prime i}=0
$$

where $\sum$ is the summation over the kicks from the 0th turn to the $n$th turn. Using the two equations above, we can solve the required vertical tune for a given number of kicks and orbit turns.

There are many possible solutions to Eq. (2). For example, we wish to cancel the displaced orbit using the second kick within two orbit turns. The orbit offsets and angles created by these two kicks (kick at the 0th and 2nd turn) at the 2nd turn are

$$
\begin{aligned}
y_{2}^{0}=\theta_{y}^{0} \beta_{y} \sin 4 \pi \nu_{y}, & y_{2}^{\prime 0}=\theta_{y}\left(\cos 4 \pi \nu_{y}-\alpha_{y} \sin 4 \pi \nu_{y}\right), \\
y_{2}^{2}=0, & y_{2}^{\prime 2}=\theta_{y}^{0} .
\end{aligned}
$$
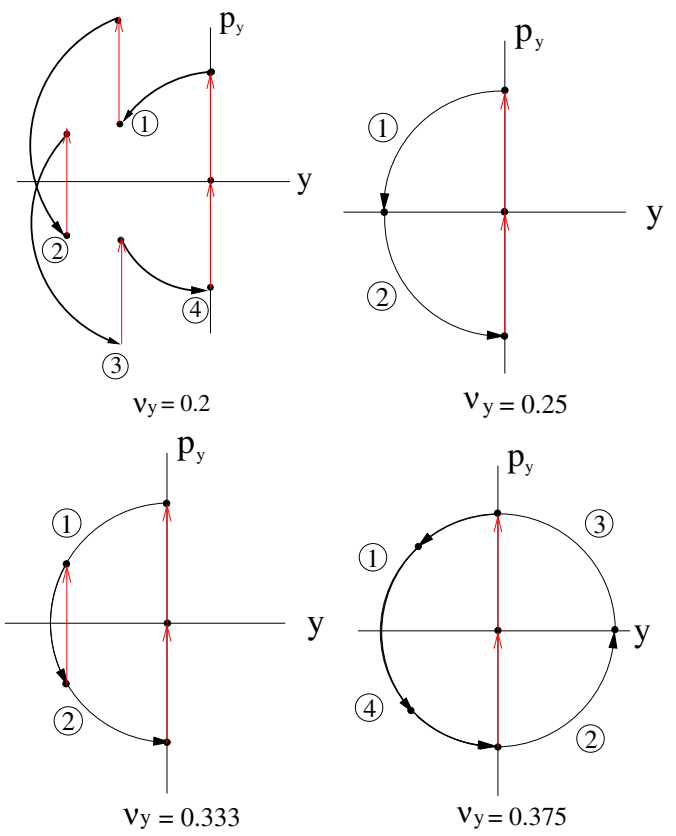

FIG. 1. Illustration of KAC schemes in a normalized phase space at different tunes.

Applying Eq. (2), we can solve that the required vertical tune $\nu_{y}=0.25$. This is the simplest stable solution. We may also cancel the displaced orbit using the second kick within one turn. But the required tune is at a half integer $\nu_{y}=0.5$ at which the machine becomes unstable.

Figure 1 illustrates the KAC scheme in a normalized phase space at different machine tunes of $0.2,0.25,0.333$ and 0.375 . For example, at tune $\nu_{y}=0.25$, the camshaft bunch is first displaced to a different vertical orbit and then proceeds two turns. At the end of the second turn, the bunch arrives at the same location but with an inverse angle to the one after the first kick. At this time, if another identical kick is applied, the bunch will be put back to its original orbit.

After the beam orbit is restored, we can wait any time of period and then repeat this kick and cancel process, thus creating single-bunch pulses with adjustable repetition rates. This kick-and-cancel process in time domain is illustrated in the Fig. 2. On each pass around the ring, the camshaft kicker either kicks or not by the same amount of kick angle $\theta_{y}$. In doing this one creates a kick-and-cancel pattern that repeats every $\mathrm{N}$ turn, where we call $\mathrm{N}$ the KAC period. As illustrated in Fig. 2, the kick-and-cancel pattern is " 10100 " and repeats every 5 turns, where " 1 " represents kick, and " 0 " represents no kick. The KAC period for this example is 5 orbital turns.

\section{Closed orbit}

As discussed above, the electron beam is periodically displaced by the kicker. This orbit displacement is actually a stable closed orbit distortion. To prove this we first need to construct a large multiturn ring with the small ring 


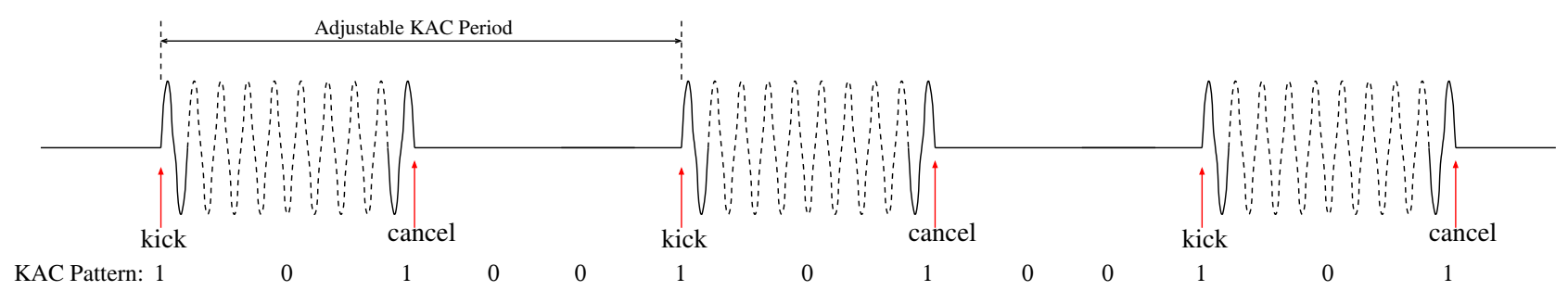

FIG. 2. Illustration of the KAC scheme in time domain. The KAC pattern is " 10100 " and KAC period is 5 orbital turns.

according to the KAC pattern. The number of the small rings made up of the large ring is given by the $\mathrm{KAC}$ period $N$. Let us look at the example with the vertical tune $\nu_{y}$ of 0.25 as shown in Fig. 3. In this example, the KAC pattern is 1010000 and the KAC period is seven orbital turns, therefore there are total seven small rings in this large multiturn ring and two small rings with kicker on. The ring with kicker on is labeled with "1", others labeled with " 0 ". Since the vertical tune of the ring is 0.25 and there are two turns between the first-kick and cancel-kick, the transfer matrix $R_{1}$ from the first-kick to the cancel-kick is a negative identity $-I$.

Let us now solve the closed orbit for this KAC scheme at tune $\nu_{y}=0.25$. To have the closed orbit just before the first kick, the following equation needs to be met

$$
\left(\begin{array}{c}
y_{\mathrm{co}} \\
y_{\mathrm{co}}^{\prime}
\end{array}\right)=R_{2} \cdot R_{k} \cdot R_{1} \cdot R_{k}\left(\begin{array}{c}
y_{\mathrm{co}} \\
y_{\mathrm{co}}^{\prime}
\end{array}\right)
$$

where $R_{2}$ is the transfer matrix from the cancel kick to the beginning of the first kick, $R_{k}$ is the transfer matrix of the kick. Assuming the kick is a thin dipole kick which only changes the beam angle and using $R_{1}=-I$, we can have

$$
\left(\begin{array}{c}
y_{\mathrm{co}} \\
y_{\mathrm{co}}^{\prime}
\end{array}\right)=R_{2} \cdot R_{1}\left(\begin{array}{c}
y_{\mathrm{co}} \\
y_{\mathrm{co}}^{\prime}
\end{array}\right)
$$

i.e.,

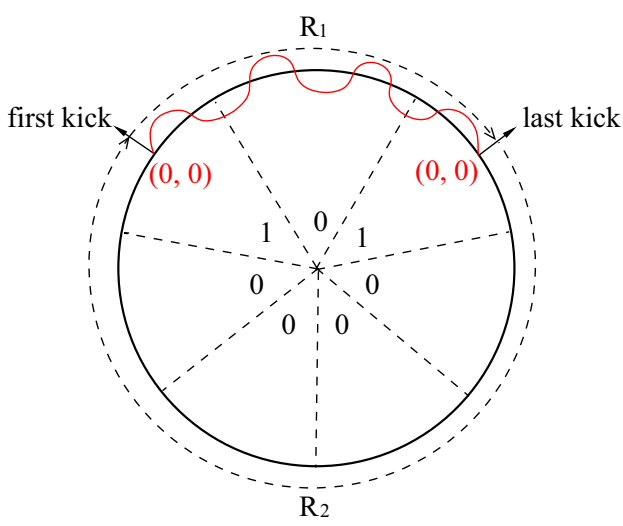

FIG. 3. A large multiturn ring. The vertical tune of the small ring is 0.25 . The KAC pattern is KAC101000. Seven rings are concatenated in this example. Two rings with kicker turn-on are labeled with " 1 ", other rings with kicker off are labeled with " 0 ".

$$
\left(R_{2} \cdot R_{1}-I\right)\left(\begin{array}{c}
y_{\mathrm{co}} \\
y_{\mathrm{co}}^{\prime}
\end{array}\right)=0
$$

where $R_{2} \cdot R_{1}$ is just the one turn map of the large multiturn ring. Equation (6) is a homogeneous linear equation system. It either has a zero solution or an infinite number of solutions, which is determined by the determinant of the matrix $\left|R_{2} \cdot R_{1}-I\right|$, i.e.,

$$
\left(\begin{array}{c}
y_{\mathrm{co}} \\
y_{\mathrm{co}}^{\prime}
\end{array}\right)= \begin{cases}0 & \text { if }\left|R_{2} \cdot R_{1}-I\right| \neq 0 \\
\text { any } & \text { if }\left|R_{2} \cdot R_{1}-I\right|=0 .\end{cases}
$$

Assuming the transfer matrix $R_{2}$ from the cancel kick to the first kick is

$$
R_{2}=\left(\begin{array}{cc}
E & F \\
G & H
\end{array}\right)
$$

and using $R_{1}=-I$ and $\left|R_{2}\right|=1$, we can have

$$
\left|R_{2} \cdot R_{1}-I\right|=2-(-E-H) .
$$

Here, $-E-H$ is just the trace of the one turn map of the multiturn ring, i.e., $-E-H=\operatorname{Tr}\left(R_{2} \cdot R_{1}\right)=2 \cos \left(2 \pi N \nu_{y}\right)$, where $N$ is the KAC period and $\nu_{y}=0.25$ is the vertical tune. Therefore, we can have

$$
\left(\begin{array}{c}
y_{\mathrm{co}} \\
y_{\mathrm{co}}^{\prime}
\end{array}\right)= \begin{cases}0 & \text { if } N / 4 \neq \text { integer; } \\
\text { any } & \text { if } N / 4=\text { integer. }\end{cases}
$$

It is now clear that if $N / 4$ is not an integer, the closed orbit $\left(y_{\mathrm{co}}, y_{\mathrm{co}}{ }^{\prime}\right)$ just before the first kick can be found and equal zero. Therefore, the oscillation of the kicked beam is localized between the first kick and the cancel kick, i.e., the KAC orbit has zero displacement outside of the kicks and nonzero displacement between the kicks as shown in Fig. 3. The KAC beam orbit is a stable closed orbit. If $N / 4$ is an integer, $\left(y_{\mathrm{co}}, y_{\mathrm{co}}{ }^{\prime}\right)$ can be any value, which means the $\mathrm{KAC}$ orbit is unstable. We need to avoid this resonance condition in order to have a stable KAC operation. In general, this integer resonance condition exists at other tunes. We know that the closed orbit distortion (COD) of the large multiturn ring is proportional to $\frac{1}{\sin \pi N \nu_{y}}$. As $N \nu_{y}$ approaches an integer, the COD diverges and the ring 


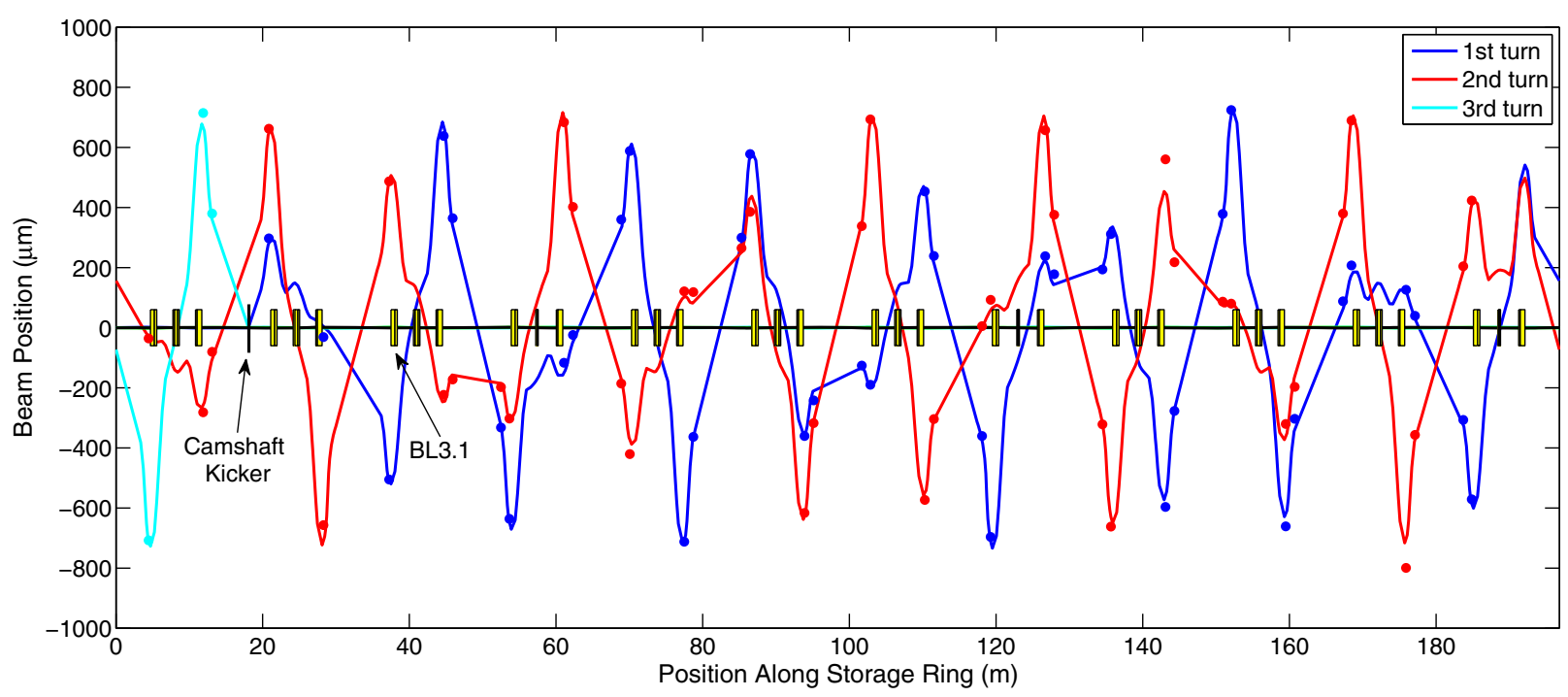

FIG. 4. Closed orbit of the KAC beam. The solid curves represent the simulation results and dots represent turn-by-turn BPM measurements.

become unstable. In this case, the KAC orbit is not well defined.

At a stable closed orbit condition, the KAC orbit can be calculated by evaluating the closed orbit of the large multiturn ring using an accelerator modeling code, such as AT, ELEGANT or MAD. Figure 4 shows the calculated KAC orbit for the ALS storage ring at tune $(16.16,9.25)$ with the vertical kick angle of $73 \mu \mathrm{rad}$. We can see that the first kick puts the beam to a displaced orbit for two turns, and then the cancel-kick restores the displaced orbit for the rest of turns. There are three beam orbit at each location around the ring, two are displaced orbits and one is the design orbit. The orbit offsets are different at different locations. For example, at synchrotron radiation diagnostic beamline 3.1, the orbits are displaced for about $500 \mu \mathrm{m}$ on both side of the designed orbit.

\section{Resonance}

As discussed above, the KAC orbit becomes unstable as $N \nu_{y}$ approaches an integer. In addition, to have a stable PSB-KAC operation, we also need to avoid the following coupling resonances

$$
m_{x}\left(N \nu_{x}\right)+m_{y}\left(N \nu_{y}\right)=n,
$$

where $m_{x}, m_{y}$ and $N$ are integer, $N$ is the KAC period, $N \nu_{x}$ and $N \nu_{y}$ are the horizontal and vertical tunes of the large multiturn ring.

To study these resonance conditions, turn-by-turn tracking is carried out at different KAC period $N$ from 3 to 100 for the ALS storage ring lattice at tune $(16.16,9.25)$. The beam orbit is monitored at the beam line 3.1. As expected, the KAC orbit becomes unstable when the KAC period is an integer multiple of 4 , such as $N=4,8,12,16$ and so on. Besides that, the beam becomes unstable at the KAC period $N=10,22,2539,50,61,66,75,78$ and 89 . Figure 5 shows some examples of simulated TbT orbits at the KAC period $N=5,8,22,39$. If the KAC period is away from the resonance $N=5$, the TbT orbit is very stable as shown in Fig. 5(a). However, at the integer resonance $N=8$, the TbT orbit becomes unstable as shown in Fig. 5(b). At the KAC period $N=22$ and 39, the tune of the large multiturn ring are $(355.52,203.50)$ and $(630.24$, $360.75)$, respectively. Both of them are near the coupling resonance. Therefore, the KAC orbits become unstable as shown in Fig. 5(c) and (d). At these resonances, however we can stabilize the beam orbit by slightly adjusting the horizontal tune away from the resonance. The stabilized KAC orbits after adjusting horizontal tunes are shown Fig. 5(e) and (f).

\section{E. Decoherence and stability}

Besides the resonances, the KAC beam orbit can also be affected by other factors such as the reproducibility of the kick, variation of the machine tune, tune shift with amplitude and chromaticity. If not well controlled, these effects can cause unstable KAC orbits.

The reproducibility of the kick amplitude depends on the stability of the power supply. Measurements using an analog oscilloscope show that the relative fluctuations are approximately $2 \times 10^{-3}$, which gives rise to about $0.5 \mu \mathrm{m}$ vertical orbit motion. It is only $5 \%$ of the vertical beam size (about $10 \mu \mathrm{m}$ ), which can be neglected.

To restore the displaced orbit, the machine tune needs to be at a right value and stable. The machine tune variation or shift can cause the residual oscillation of the KAC beam. This residual oscillation amplitude can be estimated by $\Delta y=2 \pi \beta_{y} \theta_{y} \Delta \nu_{y}$, where $\beta_{y}$ is the beta function at the 


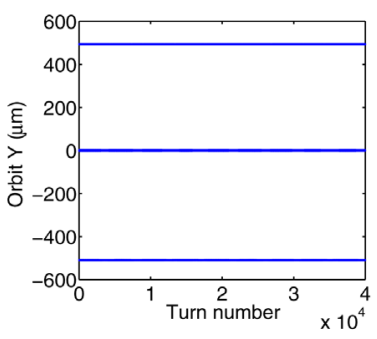

(a) $\mathrm{N}=5, \nu_{\mathrm{x}}=16.16$

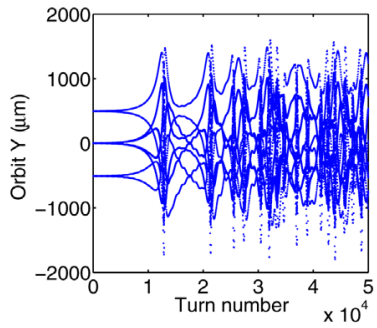

(c) $\mathrm{N}=22, \nu_{\mathrm{x}}=16.16$

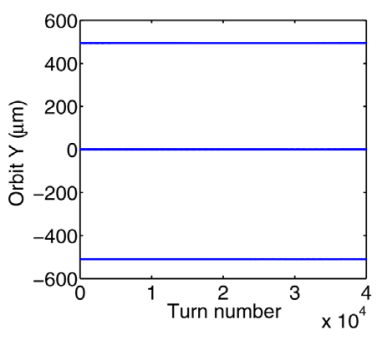

(e) $\mathrm{N}=22, \nu_{\mathrm{x}}=16.167$

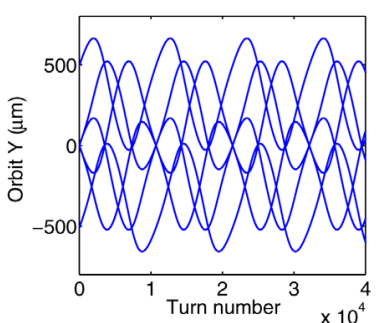

(b) $\mathrm{N}=8, \nu_{\mathrm{x}}=16.16$

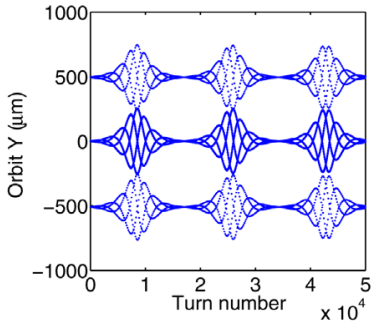

(d) $\mathrm{N}=39, \nu_{\mathrm{x}}=16.16$

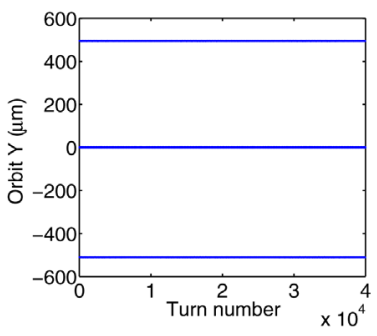

(f) $\mathrm{N}=39, \nu_{\mathrm{x}}=16.164$
FIG. 5. Simulated turn-by-turn beam orbits at beamline 3.1 for different KAC period $N=5,8,22,39$. (a) Stable KAC beam orbit at $N=5$; (b) Unstable beam orbit at the resonance of the integer multiple of 4, $N=8$; (c) and (d) Unstable beam orbits at the coupling resonances $N=22$ and $N=39$, respectively; (e) and (f) Stabilized beam orbits after adjusting the horizontal tune away from the coupling resonances at $N=22$ and $N=39$, respectively.

kicker location, $\theta_{y}$ is the kick angle and $\Delta \nu_{y}$ is the tune variation. There are three major causes of the tune variation or shift, the machine variation (power supply fluctuations and insertion device movements), tune shift with amplitude and chromaticity effect. The vertical tune shift due to the gap changes of insertion devices greatly depends on the type of the devices and can reach to the value of about 0.02 during the normal operation at the ALS. A tune feedback and a feedforward systems have been implemented to stabilize the tune variation level to be less than 0.0002 . At the ALS, the vertical tune shift with amplitude is about $0.008 / \mu$-rad. The $73 \mu \mathrm{rad}$ kick can cause about $0.02 \mu$-rad action amplitude. Therefore the tune shift due to the kick is about 0.00016 . The third cause is the chromaticity effect which could shift the betatron tune of the beam when its energy deviates from the reference energy. At the ALS, the vertical chromaticity is about 1.4 and the RMS beam energy spread is about $9.5 \times 10^{-3}$ which gives the tune shift of about 0.0013. Overall, the total tune shift and variation due to the above three factors is about 0.0013, which could result in about $1 \mu \mathrm{m}$ beam motion, which is negligible at ALS. In summary, these estimates indicate that for the ALS parameters the residual beam orbit motion and beam size increase for the KAC scheme should be acceptably small.

In addition, the kicked camshaft bunch may affect the transverse multibunch instabilities including the resistive wall instability and ion instabilities since it oscillates on a different closed orbit. These instabilities have been investigated in the paper [8]. It appears that the orbit offset and tune shift due to the kick does not contribute to any resistive wall instability. On the other hand, it can help reduce the possibility of ion trapping and weaken the instability since the kicked orbit can drive the ions to a large amplitude. At the ALS, we have not observed any transverse multibunch instabilities during the KAC operation.

\section{SETUP}

\section{A. Machine setup}

To run the PSB-KAC scheme, we first need to setup the storage ring lattice at a correct working tune. At the ALS, the nominal tunes of the machine was $(16.25,9.20)$ after the recent brightness upgrade [9]. To run the KAC scheme at the vertical fractional tune of 0.25 , we need to adjust the vertical tune from 9.20 to 9.25 . Although the horizontal tune is not required by the KAC scheme, it should be determined by the lattice performance. To find a good working point for the KAC operation, a scan of the horizontal tune was carried out and $(16.16,9.25)$ was found to be a good working point. To integrate the KAC scheme to the normal operation, the ALS operational lattice has been successfully migrated to the pseudosingle bunch lattice at the tune of $(16.16,9.25)$.

Tune feedback has also been implemented during the lattice migration. With this feedback on, we are able to stabilize the machine tune variation at the level of 0.0002 . This is a critical requirement to maintain a stable PSB-KAC operation.

\section{B. Kicker timing setup}

As mentioned before, we do not have the capability to change the kick amplitude and polarity in a fast way, but we have the flexibility to adjust the kick frequency and pattern. This allows us to operate the camshaft kicker at different KAC schemes. Figure 6 shows the schematic of the pulser timing setup for the KAC scheme at tune of 0.25 . The external trigger signal can be provided by either beamline users or accelerator operators. If the trigger signal comes from the accelerator operators, the signal is scaled from the Storage Ring Orbit Clock (SROC) using a Stanford Digital Delay Generator. This trigger signal is fed into the Agilent Arbitrary Waveform Generator to generate two burst pulses. The pulse width is slightly less than one orbital 


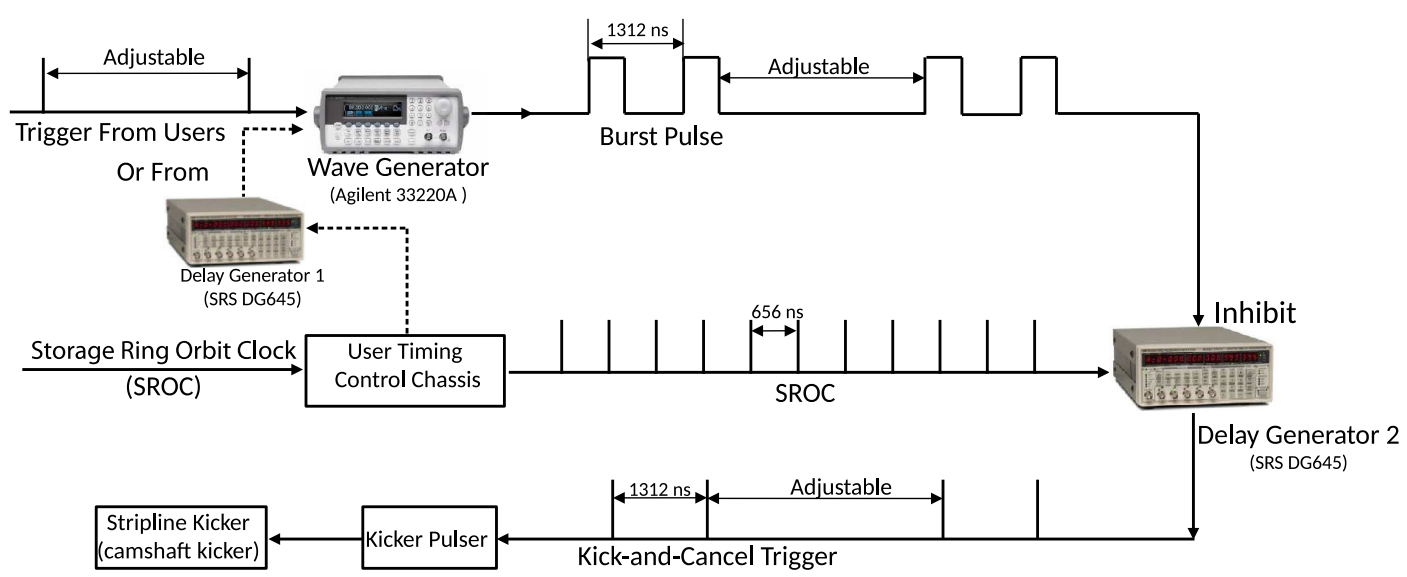

FIG. 6. Schematic of pulser timing setup for the kick-and-cancel operation at tune 0.25

period $656 \mathrm{~ns}$ of the storage ring. The distance between two burst pulses is about two orbital turns 1312 ns. These burst pulses are then fed into another Stanford Digital Delay Generator to inhibit the SROC signals. Thus, after the inhibition a KAC signal consisting of two SROCs is created and these two SROCs are separated by two orbital turns. This KAC signal is then used to trigger the kicker pulser amplifier which further drives the camshaft bunch kicker. The repetition rate of this KAC signal can be easily adjusted by controlling the repetition rate of the external trigger signal.

The advantage of this timing setup is that the synchronization between the kicks and the electron bunches is independent of the external trigger signal. This synchronization is realized by tuning either the User Timing Control Chassis or the second Stanford delay Generator. Thus, users have the flexibility to change the timing of their trigger signal without affecting the camshaft kicker timing and KAC beam stability.

Figure 7 shows the monitored kick pulse and camshaft bunch signal. Since the ion cleaning gap is $66 \mathrm{~ns}$ at the ALS and the full width of the kick pulse is about $70 \mathrm{~ns}$ with a

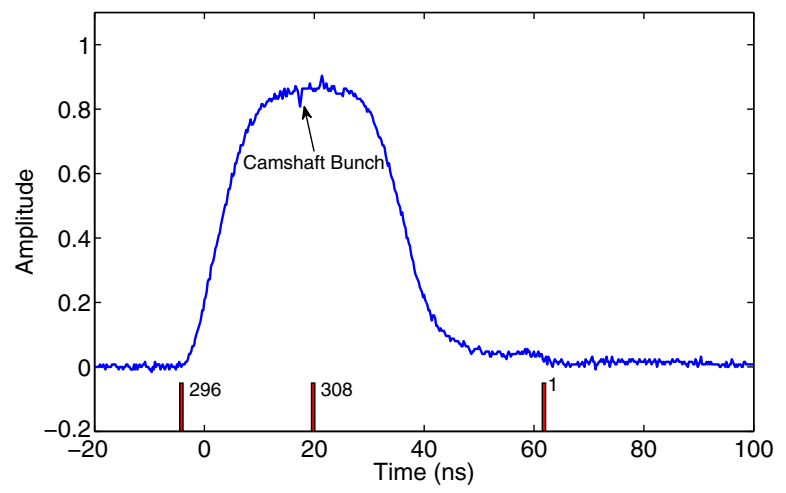

FIG. 7. Measured kicker pulse and camshaft bunch signals. The position of buckets 296, 308 and 1 are labeled in the plot. The camshaft bunch is filled at bucket 308 . long tail, the camshaft bunch is filled at the bucket 308 to minimize the kick perturbation on the main bunch train. The camshaft bunch is timed to the center of the pulse peak by adjusting the delay between the SROC and the electron bunch via User Timing Control Chassis.

\section{MEASUREMENTS AND DIAGNOSTICS}

At the ALS, various tools and instruments have been utilized to study the pseudosingle bunch kick-and-cancel operation mode, including the turn-by-turn ( $\mathrm{TbT}$ ) beam position monitor (BPM), fast-gated and non-gated CCD cameras and bunch-by-bunch transverse feedback system. Each instrument can provide different study aspects on the PSB-KAC, and allows us to better understand this operational mode. In the following, we are going to present the PSB-KAC studies with these tools and instruments.

\section{A. TbT BPM measurements}

There are 44 state-of-art TbT BPMs newly installed at the ALS around the storage ring. These BPMs provide a valuable tool to study the KAC scheme in turn-by-turn bases. Here, two examples are presented.

With these BPMs, we are able to measure the KAC beam closed orbit around the ring. The measured results are shown in Fig. 4. A good agreement between the measurement and simulation is observed. For this measurement, a $5 \mathrm{~mA}$ single camshaft bunch is filled in the ring. The kick angle is $73 \mu \mathrm{rad}$ and KAC frequency is $1 \mathrm{kHz}$. Only two turn orbits after the first kick are presented in the plot.

Figure 8 shows the TbT BPM signal at the beam line 3.1 for different KAC frequency. For these measurements, a $5 \mathrm{~mA}$ single camshaft bunch was filled in the storage ring. As shown in the inserted plot of Fig. 8(c), the beam orbit is displaced for two turns after the first-kick then immediately restored to the design orbit after the cancel kick. This orbit displacement is repeated at frequency (a) $4 \mathrm{kHz}$, (b) $2 \mathrm{kHz}$ and (c) $1 \mathrm{kHz}$ which can be easily adjusted by beam users. 


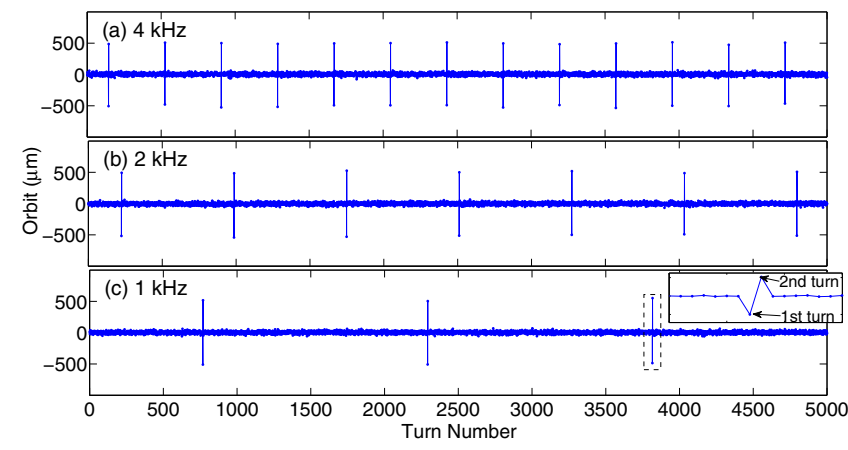

FIG. 8. TbT BPM signal at beam line 3.1 for different KAC frequency (a) $4 \mathrm{kHz}$, (b) $2 \mathrm{kHz}$, and (c) $1 \mathrm{kHz}$. The inserted in (c) shows the magnified beam orbit around one KAC pulse.

\section{B. Camera measurements}

At the ALS, the electron beam in the storage ring is routinely monitored at our synchrotron radiation diagnostic beam line 3.1 [10]. At this beam line, the synchrotron light emitted from the bend magnet is focused by a Kirkpatrick-Baez $(\mathrm{KB})$ mirror pair onto a image plane where a Bismuth/Germanium-Oxide (BGO) crystal converts the focused light to visible light. This visible light image is magnified by an optical microscope and recorded by a CCD camera. This diagnostic system has been in the operation for more than 20 year since the ALS commissioning. At this beam line, the KAC orbits are displaced for about $500 \mu \mathrm{m}$ on both side of the designed orbit as shown in Fig. 4. It is an ideal location to study the KAC scheme. To have a better diagnostic capabilities for the KAC studies, we recently upgraded the endstation of this beamline. The slow response BGO crystal (about $300 \mathrm{~ns}$ ) was replaced by a fast response (about $40 \mathrm{~ns}$ ) LYSO crystal (Cerium-doped Lutetium Yttrium Orthosilicate). We also redesigned the old optical microscope, replaced the old CCD camera by a new nongated camera (Manta G-145, ALLIED Vision Technologies) and installed a new fastgated camera (PI-MAX4:1024i, Princeton Instruments). A beam splitter is used in the redesigned optical microscope to allow operating the nongated and fast-gated cameras at the same time. The fast-gated camera has a very fast gating rate, capable of measuring the beam image in turn-byturn bases.

Figure 9 shows the measured KAC beam image with a nongated camera. Three beam spots are clearly separated, the central one corresponding to the unkicked design orbit and other two the kicked orbits. The separation between the two kicked orbits is about $950 \mu \mathrm{m}$, which is consistent with the simulation. For this measurement, the storage ring was filled with a $5 \mathrm{~mA}$ single camshaft bunch, and the beam was kicked at the maximum kick-and-cancel frequency, i.e., the $\mathrm{KAC}$ period is 3 and there is no waiting turn between each KAC process. Therefore, the three orbits have the same repetition rate of $500 \mathrm{kHz}$ and the same mounts of current of $1.67 \mathrm{~mA}$.

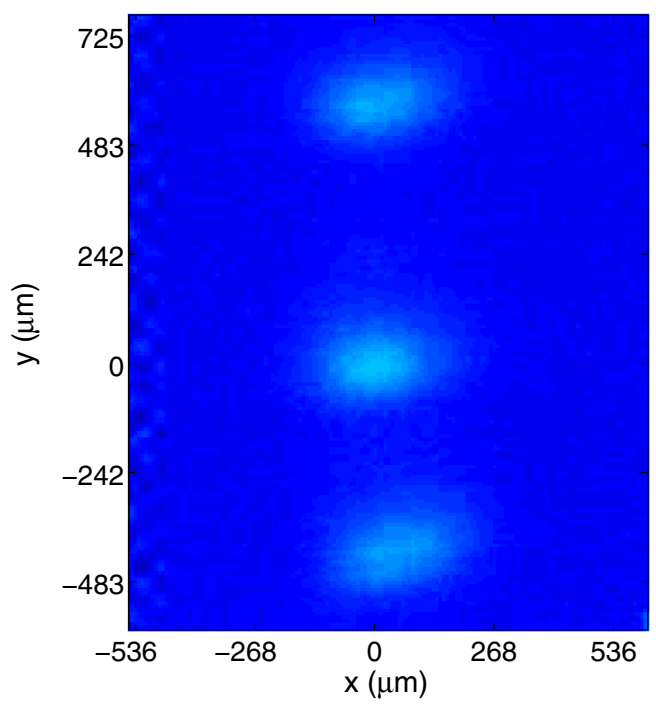

FIG. 9. Beam image at synchrotron diagnostic beam line 3.1 measured with a nongated CCD camera. For this measurement, the storage ring was filled with a $5 \mathrm{~mA}$ single camshaft bunch, and the beam was kicked at the maximum kick-and-cancel frequency.

Figure 10 shows a sequence of beam images captured using our fast-gated camera. For this measurement, a $5 \mathrm{~mA}$ single bunch is filled in the ring and the KAC frequency is $4 \mathrm{kHz}$. The camera is triggered by the kicker trigger signal. By adjusting the delay between the trigger and the camera gate opening, we are able to capture beam images at a sequence of turns after the kick. As we can see, the beam spots are well separated for two displaced turns after the first kick, and then back to the design orbit after the cancel kick. The beam size stays the same during this KAC process.

A scan was carried out to study the resonance conditions at different KAC periods from 3 to 28 as shown in Fig. 11. These beam images are captured by our fast-gated camera but operated in the integrating mode. At lower KAC periods (i.e., the high KAC frequency), the kicked beam orbits repeat at a high repetition rate. Therefore, the kicked beam spots have comparable intensity to the unkicked beam and three beam spots can be clearly seen in the images. As the KAC periods $\mathrm{N}$ increased, the intensity of the kicked beam spots becomes lower and the beam images are dominated by the unkicked beam. For most of the KAC
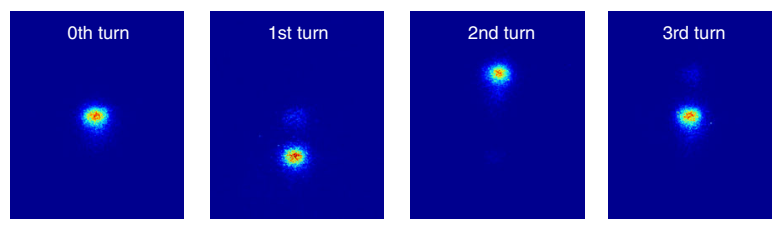

FIG. 10. A sequence of beam images at synchrotron diagnostic beam line 3.1 measured with a fast gated CCD camera at different turns after the first kick. 

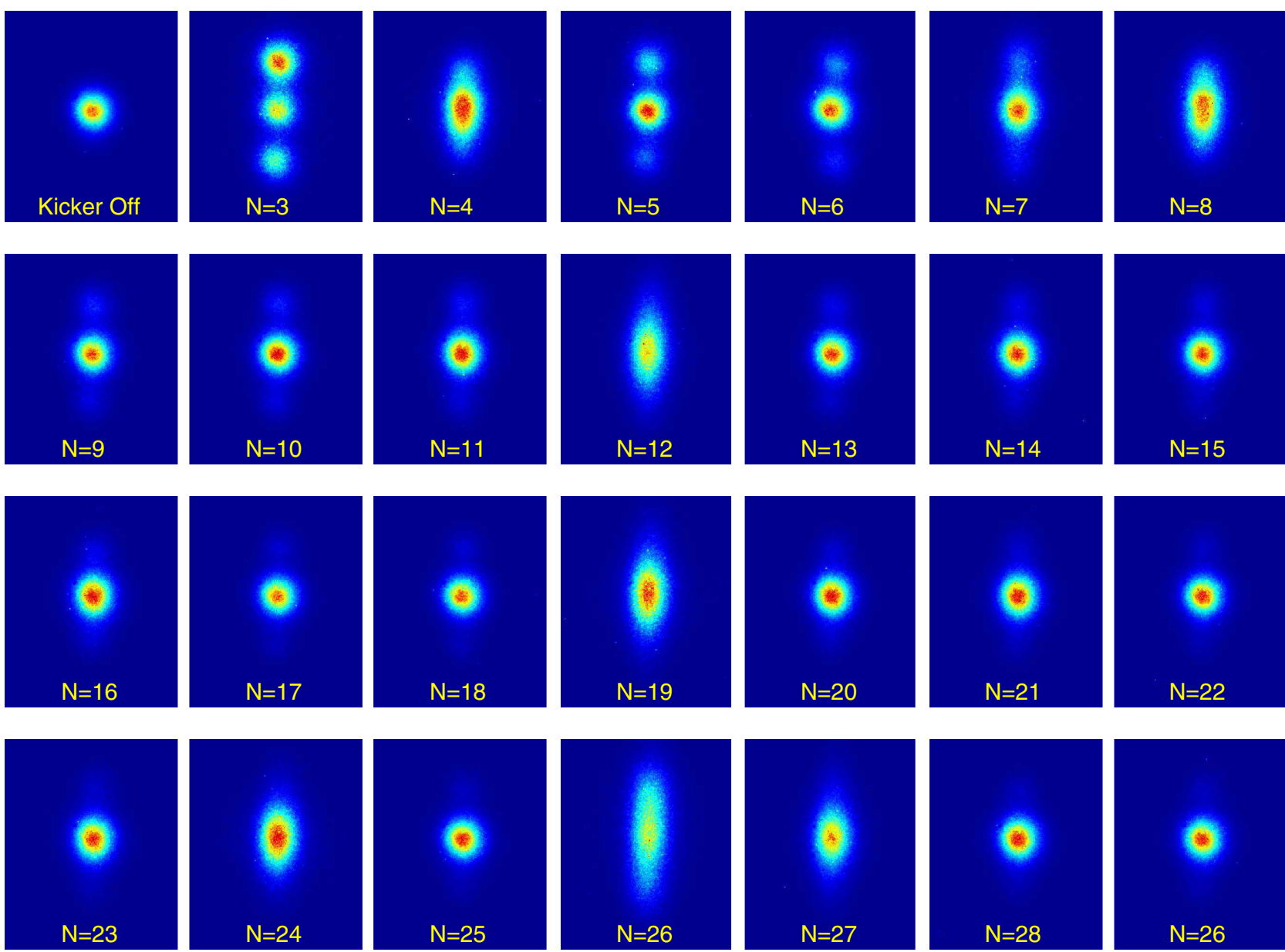

FIG. 11. Beam images measured at different KAC period N from 3 to 28. The image at the upper left corner is measured when the camshaft kicker is turned off. The lower right image is a stabilized KAC beam at $N=26$ by adjusting the horizontal tune of the machine away from the resonance.

periods, we have a stable KAC beam and the beam size is the same as one when the kicker is turned off. As expected, when approaching the resonance of the integer multiple of 4, the KAC beams become unstable. The KAC beam are also unstable at some other coupling resonances such as at $N=19,26$ and 27. However, at these resonances we can stabilize the KAC beams by slightly adjusting the horizontal tune away from the resonances. The last image (the lower right corner) in Fig. 11 shows a stabilized KAC beam at $N=26$ after the horizontal tune of the machine is adjusted away from the resonance.

\section{Diagnostics using transverse bunch-by-bunch feedback system}

During the multibunch operation, the ALS ring is usually filled with $495 \mathrm{~mA}$ multibunch train and $5 \mathrm{~mA}$ single camshaft bunch. It becomes difficult to measure this $5 \mathrm{~mA}$ camshaft bunch out of $495 \mathrm{~mA}$ bunch train using TbT BPMs and CCD cameras during this operation. Recently, we replaced our old analog transverse feedback system by a digital bunch-by-bunch system developed by Dimtel, Inc. This system has the capability to detect single bunch oscillation. Therefore, it can be used as a diagnostic tool for our PSB-KAC beam during the multibunch operation.

Figure 12 shows the multibunch waveforms panel of this feedback system with the camshaft kicker turned off and on. When the kicker is off, the RMS oscillation amplitude of the camshaft bunch at 308 has the same level as other bunches as shown in the lower left sub-panel of Fig. 12(a). When the kicker is on, the camshaft bunch oscillation is standing out. The subpanel in the upper right corner of Fig. 12(b) shows the oscillation signal of the kicked camshaft bunch in the time domain with the frequency of $4 \mathrm{kHz}$. This regular oscillation indicates that the kicked camshaft bunch is stable. If the camshaft bunch becomes unstable, we will see an irregular oscillation in this panel.

The feedback system can also be used to improve the performance of the PSB-KAC operation. For example, with this system we can increase the transverse mode coupling instability (TMCI) threshold of the single camshaft bunch therefore increasing the intensity of the PSB beam. 


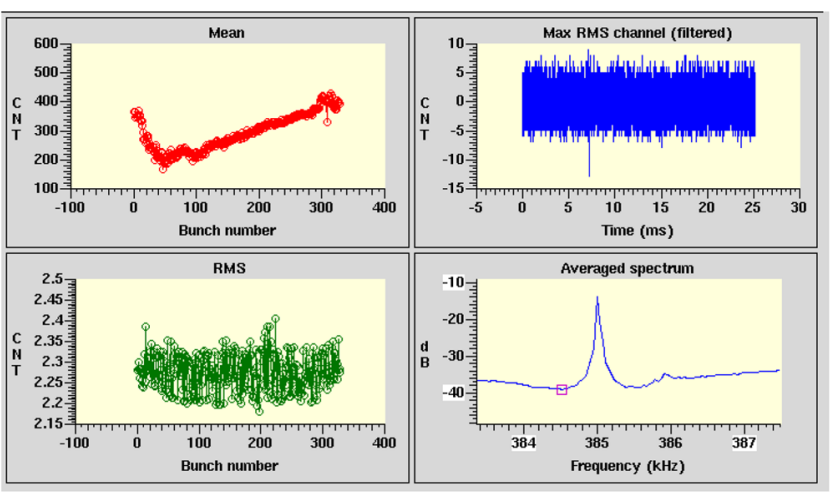

(a) Kicker Off

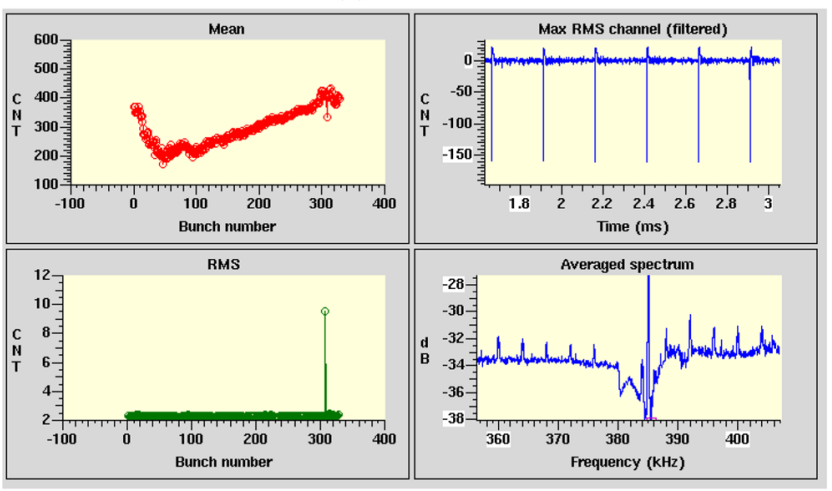

(b) Kicker On

FIG. 12. Multibunch waveforms panel of the Dimtel's transverse feedback system.

Recently we have demonstrated that we can increase the camshaft bunch current from $6 \mathrm{~mA}$ to about $15 \mathrm{~mA}$, which could improve the PSB beam intensity by a factor of 2.5 . We may also use this system to stabilize the beam when at some KAC resonances, which needs to be further explored.

\section{Tests with user beam lines}

This new PSB-KAC operation mode has been tested at different $\mathrm{X}$-ray user beam lines at the ALS. Results confirm that the radiation light emitted from the kicked camshaft bunch can be well separated from the main bunch train radiation at user beam lines and the background radiation from unwanted bunches can be significantly suppressed [3-5]. These PSB beams have been used in pump-probe measurements on spin crossover complexes, and in a warm-dense-matter (WDM) demonstration experiment using a time-integrating single-shot streak camera, achieving improved signal to noise while reducing X-ray exposure by three orders of magnitude [4]. Before brought to the normal operation, this PSB-KAC has been tested at multibunch user beamlines to study its impact on their experiments and there were no significant negative effects observed, which means that this mode is transparent to multibunch users.

\section{CONCLUSIONS}

The pseudosingle-bunch kick-and-cancel (PSB-KAC) mode has been successful brought into the user operation since 2014 at the Advanced Light Source (ALS). With this mode, it is possible to run both single-bunch and multibunch operations at the same time. This greatly expands the capabilities of the light source to satisfy multibunch and timing experiments simultaneously. With the proposed kick-and-cancel scheme, the pulse repetition rate of the PSB photon beam can be adjusted from $\mathrm{Hz}$ to $\mathrm{MHz}$, which can significantly alleviate complications of using high power choppers, substantially reduce the rate of sample damage, and greatly increase the variety and quality of experiments that can be done without using gated detectors.

\section{ACKNOWLEDGMENTS}

The authors would like to thank Slawomir Kwiatkowski, James Julian, David Plate, Ray Low and Ken Baptiste who constructed the PSB kicker and pulser. We also wish to thank the ALS management for their support and encouragement of these studies. We also would like to thank ALS beam line scientists who helped us carry out tests of this new operation mode. This work is supported by the Director Office of Science of the U.S. Department of Energy under Contract No. DE-AC02-05CH11231.

[1] G. Portmann et al., in Proceedings of the 22nd Particle Accelerator Conference, PAC-2007, Albuquerque, NM (IEEE, New York, 2007), p. 1182.

[2] G. Portmann et al., in Proceedings of the Beam Instrumentation Workshop, Tahoe City, CA, 2008, p. 313.

[3] C. Sun, G. Portmann, M. Hertlein, J. Kirz, and D. S. Robin, Phys. Rev. Lett. 109, 264801 (2012);

[4] M.P. Hertlein et al., J. Synchrotron Radiat. 22, 729 (2015).

[5] C. Sun, G. Portmann, M. Hertlein, J. Kirz, and D. S. Robin, Synchrotron Radiat. News 26, 9 (2013).

[6] S. Kwiatkowski et al., in Proceedings of the 10th European Particle Accelerator Conference, Edinburgh, Scotland, 2006 (EPS-AG, Edinburgh, Scotland, 2006), p. 3547.

[7] L. Blumberg, Brookhaven National Laboratory Memorandum, May 29, 1980.

[8] B. C. Jiang, G. X. Xia, M. Z. Zhang, S. Q. Tian, and Z. T. Zhao, Phys. Rev. ST Accel. Beams 17, 040703 (2014).

[9] C. Steier et al., J. Phys. Conf. Ser. 493, 012030 (2014).

[10] T. R. Renner, H. A. Padmore, and R. Keller, Rev. Sci. Instrum. 67, 3368 (1996). 